\title{
Interaction of a MW AM Broadcast Transmitter with a Large Crane and the Analysis of the Workers' Safety
}

\author{
$\underline{\text { Fatih Üstüner }}^{1}$ \\ ${ }^{1}$ TÜBİTAK BILGEM UEKAE \\ Gebze, Kocaeli, Turkey \\ fatih.ustuner@uekae.tubitak.gov.tr
}

\begin{abstract}
During the construction phase of a wind power plant in Turkey, an electromagnetic interaction was observed with a large crane and an AM broadcast transmitter. The interaction resulted in large current levels which poses health hazards to the workers. Initial grounding measures were not sufficient. In order to quantify the current levels, to assess the degree of the hazard and to find a practical remedy to the problem, the phenomenon is modeled and different cases with different worker configurations are analyzed. The analysis results emphasize the importance of the insulation and local grounding.
\end{abstract}

\section{Introduction}

In the construction phase of a wind power plant at Çatalca district near Istanbul, an odd phenomenon repeatedly occurred. During the assembly phase of the wind turbine tower components, assembly workers at the top of the tower component have experienced shock and burning pain while touching another incoming component carried by a large crane. The construction company suspected from atmospheric electricity and grounded the large crane with a long cable. However, the problem persisted and the company requested TÜBİTAK to carry out an electromagnetic field survey. The field survey identified the source of the problem as an AM broadcast station located $8 \mathrm{~km}$ away from the construction site. Since the problem source was of continuous nature and posed threat to the health of the workers, all construction works were stopped until finding a remedy to the problem. In order to find a practical solution, it is decided to carry out an electromagnetic analysis of the phenomenon.

\section{Field Survey and Definition of the Problem}

In the field survey, it was noted that no RF base station is present in the construction area. Before beginning the electromagnetic field measurements, all wireless devices including cell phones were shut down. Electromagnetic fields greater than $200 \mathrm{~V} / \mathrm{m}$ were measured near the hook and it was noted that the field was decreasing rapidly as going away from the hook. Moreover, electromagnetic fields around $10 \mathrm{~V} / \mathrm{m}$ were measured near the operator cabin located on the crawlers' body of the crane. In order to investigate the source of these fields, it is decided to measure the currents on the crane body. A current probe was clamped on a piece of metal located somewhere on the crane (see Figure 1a) and a spectrum analyzer is used to monitor the current spectra. At a specific frequency $(702 \mathrm{kHz})$, a very strong signal exceeding $10 \mathrm{~mA}$ was observed. When we have tuned to this specific frequency and demodulated with the AM detector, we were faced with an AM radio broadcast signal. A quick inquiry for the identification of the broadcast station revealed that the signal is emitted by a government owned MW (medium wave) AM broadcast transmitter which was located approximately $8 \mathrm{~km}$ away from the construction site. The AM broadcast transmitter has an RF output power of $550 \mathrm{~kW}$. Its antenna is a monopole having a height of $230 \mathrm{~m}$. The ground plane of the monopole antenna consists of 120 radial wires buried inside the soil. Its geographical coordinates are $41^{\circ} 11^{\prime} 03^{\prime \prime} \mathrm{N}$ and $28^{0} 30^{\prime} 44^{\prime \prime} \mathrm{E}$.

Wind turbines in the power plant consist of a tubular steel tower having 80 meters height and a rotor with three blades, each having 44 meters length (Figure 1b). For the assembly of these wind turbines, a large crane is used (Figure 1c). The crane consists of a very long main boom, a relatively short auxiliary boom and the crawlers' body. The main boom length of the crane is $110 \mathrm{~m}$ long.

As can be easily understood, the electromagnetic wave emitted by the AM broadcast transmitter induces currents on the crane. Since the frequency of the station is $702 \mathrm{kHz}$, the corresponding wavelength is $427 \mathrm{~m}$ which is approximately four times the crane boom length. In other words, the crane acts like a quarter wavelength monopole 
antenna. It effectively captures and re-radiates the electromagnetic energy. When the assembly worker touches the component which is hanged on the hook, he becomes a part of the antenna structure and currents flow over his body. These currents cause the sensation of shock and burns. According to previous studies, the adverse effects of the current at MW frequencies consist of both electro-stimulation and heating [1]. The hazard to the worker depends on the current level. Although the current levels do not pose fatal effect, sensing shock and burning effects at 80 meters height aggravates the working conditions and disturbs the stability of the worker. According to Chatterjee et al., a male human being perception of the current by finger contact is around $40 \mathrm{~mA}$ at $702 \mathrm{kHz}$ and he senses pain when this level exceeds $50 \mathrm{~mA}$ [2]. According to IEEE C95.1 standard, the limit for contact touch current is given as $16.7 \mathrm{~mA}$ and this limit is relaxed to $50 \mathrm{~mA}$ if the exposed person is aware of the situation (controlled environment case) [1].

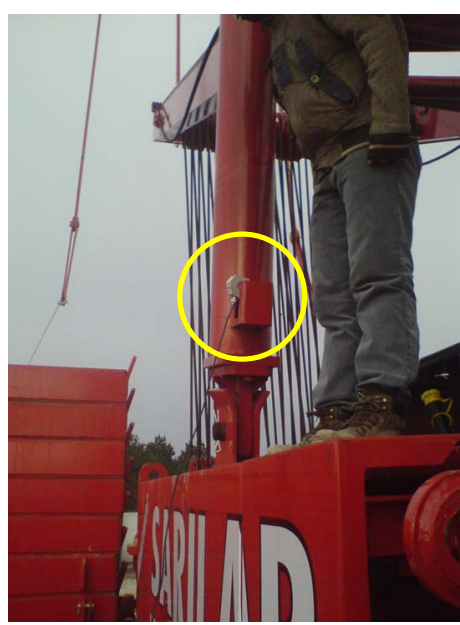

(a)

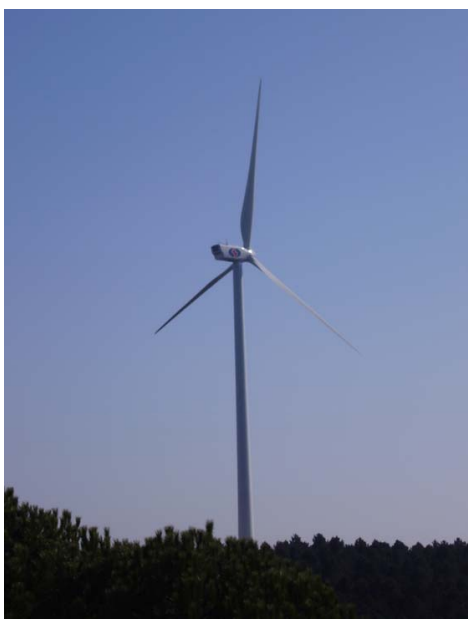

(b)

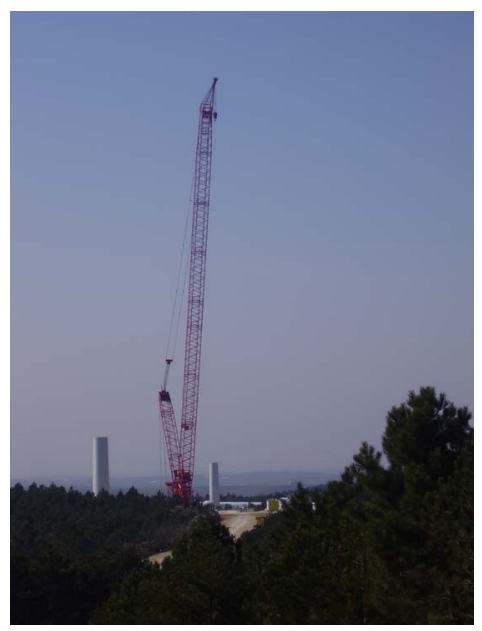

(c)

Figure 1 Current Probe Location on the Crane (a), Wind Turbine (b), Large Crane (c)

In order to investigate the current level that the worker may be exposed, a model of the situation is developed around an assembly scenario. The wind turbine tower consists of three sections. The first section placed on the ground is 30 meters long. Another 30 meter long section, the second section, is placed on top of the first section. Finally the third section having a length of 20 meters constitutes the last section of the wind turbine tower. According to the assembly scenario, the worker is working at the second level of the wind turbine tower while touching the third section which is carried by the crane. The model of the scenario can be seen on Figure 2. Three cases are of interest. In the first initial case, the hook is not grounded. Then, in the second case, a long cable is used to ground the hook. In the third and final case, a short cable is used to connect the third and second sections acting as a local ground. The crawlers' body is also grounded in all cases. The problem is to find the current flowing through the worker's body and to compare this current with the current limits given in the standards and previous studies.

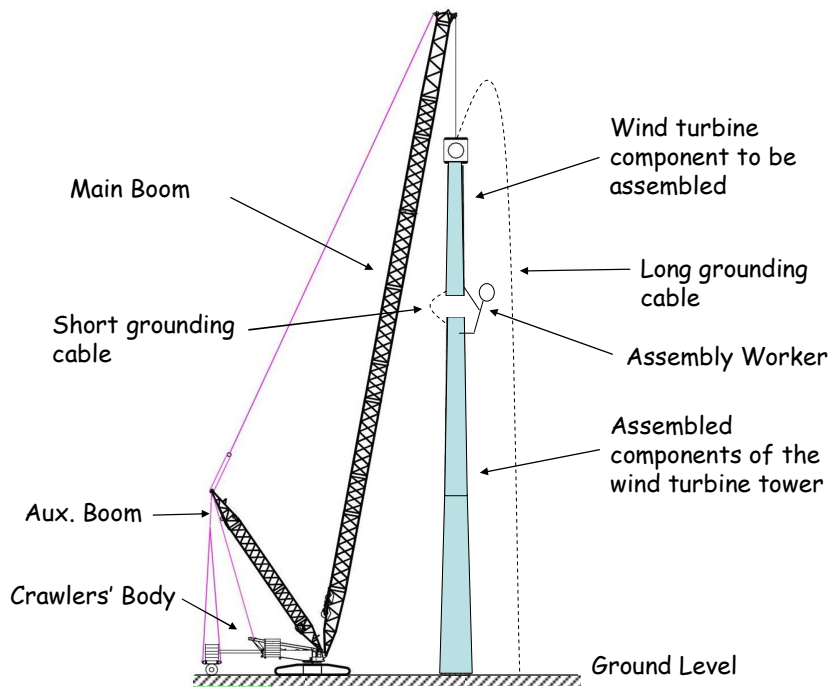

Figure 2 Assembly Scenario 


\section{Modeling and Simulation}

The problem geometry is electromagnetically modeled and analyzed using method of moments (MoM) technique. The AM broadcast transmitter antenna is completely modeled in NEC4 MoM code which is capable of modeling buried wires [3]. To simulate the earth surface, the electrical parameters of the ground is chosen as that of the wet ground given in ITU Recommendation P527-3 (conductivity $\sigma=10 \mathrm{mS} / \mathrm{m}$, dielectric permittivity $\varepsilon_{\mathrm{r}}=30$ )[4]. Approximately 3000 segments (each having $10 \mathrm{~m} \mathrm{long}$ ) are used to model the antenna. The model of the antenna and the resulting radiation pattern are seen on Figure 3.
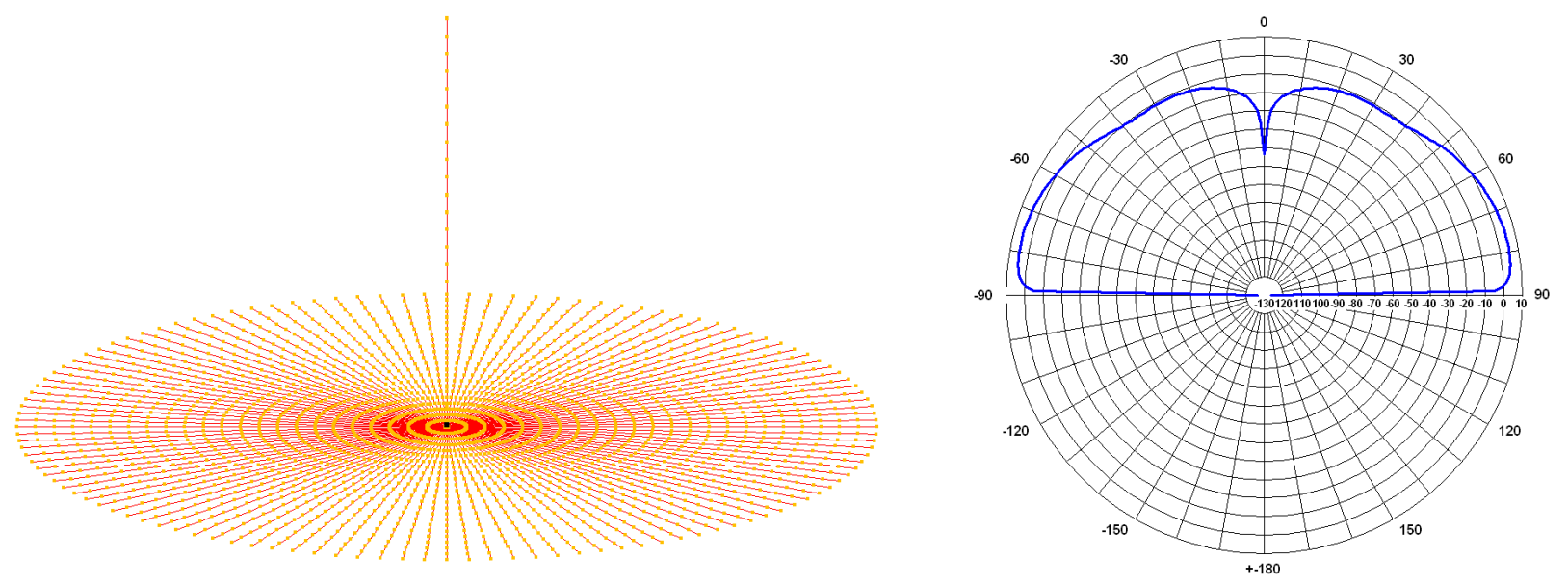

Figure 3 MoM Model of the Broadcast Antenna and its Elevation Radiation Pattern

To model the booms of the crane, a cage model having $10 \mathrm{~m}$ long segments with radius $10 \mathrm{~cm}$ is developed. The crawlers' body is grounded to the earth. Three different cases are simulated. Theses cases are given below:

- $\quad$ Case A: Hook of the crane is not grounded, the worker is between the second and third sections.

- $\quad$ Case B : Hook of the crane is grounded to the earth with a long cable, the worker is between the second and third sections.

- $\quad$ Case $\mathrm{C}$ : Hook of the crane is not grounded and the worker is between the second and third sections. A two meter long parallel wire is present with the second and third sections acting as a local ground.

These cases are illustrated in Figure 4. In the MoM model, the distance between the transmitter antenna and the crane is kept as $8 \mathrm{~km}$ as in the real life. Three different configurations of the worker are investigated. These are:

- $\quad$ Configuration \#1: Worker is at contact with tower sections with bare hands and feet.

- Configuration \#2: Worker is at contact with tower sections with bare hand but with insulated footwear.

- Configuration \#3 : Worker is at contact with tower sections with insulated gloves and insulated footwear.

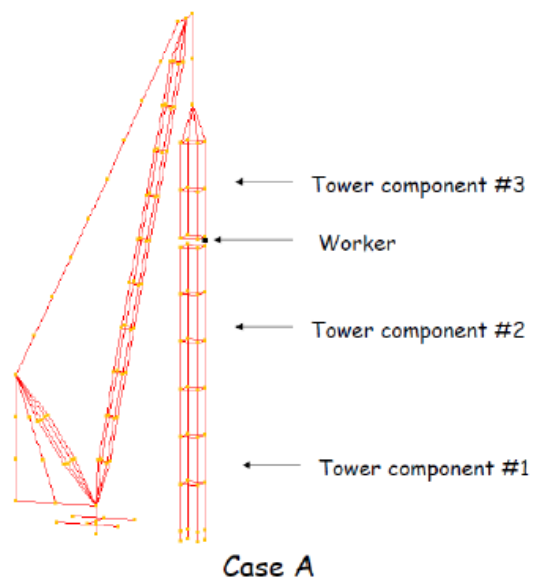

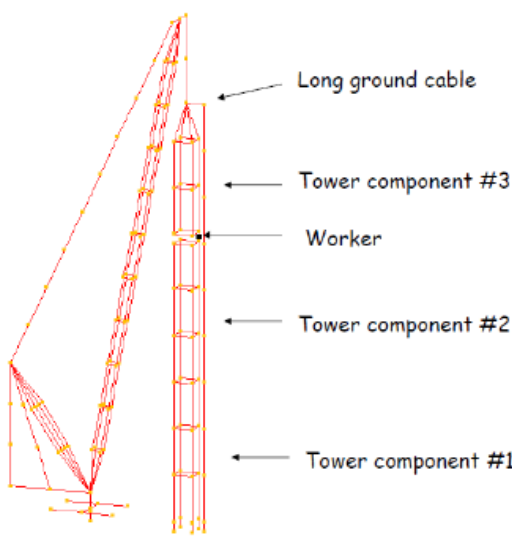

Case B

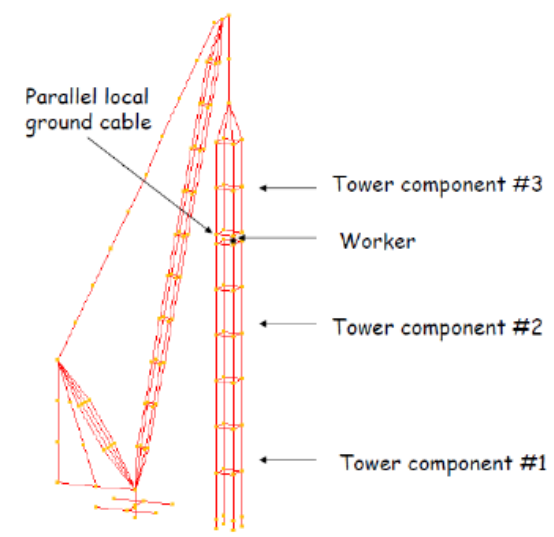

Case C

Figure 4 Different Simulation Cases 
In each configuration, the worker is modeled with lumped elements which are valid at the simulation frequency [2,5]. In the first configuration, the worker is modeled as a series connection of $430 \mathrm{ohm}$ resistor and $10 \mathrm{uF}$ capacitor. In the second configuration, the model capacitance decreases to $60 \mathrm{pF}$. In the third configuration, the capacitance decreases to $20 \mathrm{pF}$ and the model consists of a series connection of $430 \mathrm{ohm}$ resistor and $20 \mathrm{pF}$ accordingly. These lumped elements are used to load a 2 meter long MoM segment having a diameter of $40 \mathrm{~cm}$.

Then all cases in all configurations are simulated and the current level on the segment modeling the worker body is determined in each case and configuration. The simulation results are given in Table 1 . As seen from the table, the highest current level is obtained in Case A with the configuration \#1. The current level decreases approximately $80 \%$ when the worker has insulated footwear and it decreases further when the worker has gloves and footwear as the insulation medium between the worker and the metal conductors. However, the current is still comparable with the contact touch current limit of IEEE C95.1 recommendation. The long grounding cable does not reduce much the current level. This is understandable if we think the ground cable as a shorted transmission line. Below the first resonance, a shorted transmission line consists of a series connection of a resistor and an inductor. The impedance of the shorted transmission line increases with the frequency and it becomes an open circuit when its electrical length equals to quarter wavelength as it is observed in Case B. On the other hand, a short grounding cable acting as a local ground between the tower components decreases the current on the worker drastically. Unless the worker does not work with bare hands and feet, he doesn't feel the current in Case C.

Table 1 Current through the Body of the Worker (Simulation Results)

\begin{tabular}{|l|c|c|c|}
\hline & Case A & Case B & Case C \\
\hline Configuration \#1 & $235 \mathrm{~mA}$ & $214 \mathrm{~mA}$ & $16 \mathrm{~mA}$ \\
\hline Configuration $\# \mathbf{2}$ & $54 \mathrm{~mA}$ & $44 \mathrm{~mA}$ & $2 \mathrm{~mA}$ \\
\hline Configuration $\# \mathbf{3}$ & $18 \mathrm{~mA}$ & $14 \mathrm{~mA}$ & $0.8 \mathrm{~mA}$ \\
\hline
\end{tabular}

\section{Conclusion}

The electromagnetic interaction between a large crane and an AM broadcast transmitter is examined. Its effect on the assembly workers are investigated using a construction scenario. The current level through the body of the worker is simulated for different grounding cases and worker configurations. The results can be summarized as follows:

- $\quad$ Long grounding cables does not offer much help in reducing the current through the worker's body.

- $\quad$ Short ground connection between the metal bodies should be used before touching any metal object.

- Insulated gloves and footwear are indispensable tools to avoid excess current levels through the worker's body.

In the use of large cranes, workers should be cautioned against the possible hazards of the nearby RF transmitters. Resonance should also be taken into account as an aggravating factor of the interaction phenomena.

\section{References}

1. IEEE C.95.1-2005, IEEE Standard for Safety Levels with Respect to Human Exposure to Radio Frequency Electromagnetic Fields, $3 \mathrm{kHz}$ to $300 \mathrm{GHz}$, IEEE, 2005

2. Chatterjee I., Wu D., Gandhi O.P., "Human Body Impedance and Threshold Currents for Perception and Pain for Contact Hazard Analysis in the VLF to MF Band", IEEE Transactions on Biomedical Engineering, Vol. BME-33, No.5, May 1986, pp. 486-494.

3. Burke, G. J., Numerical Electromagnetics Code - NEC-4, Method of Moments, Part I: User's Manual, Lawrence Livermore National Laboratory, USA, January 1992

4. ITU Recommendation P527-3, Electrical characteristics of the surface of the Earth, ITU, 1992

5. Kanal H., Chatterjee I., Gandhi O.P., "Human Body Impedance for Electromagnetic Hazard Analysis in the VLF to MF Band”, IEEE Transactions on Microwave Theory and Techniques, Vol. MTT-32, No.8, August 1984, pp. 763-772 